\title{
Standardization of Hydropriming Duration for Enhance Seed Yield and its Quality Parameters in Chickpea (Cicer arietinum L.)
}

\author{
Sarvjeet*, S.C. Vimal and Pankaj Kumar \\ Department of Genetics and Plant Breeding, N. D. University of Agriculture and Technology, \\ Kumarganj-224 229, Faizabad (U.P.), India \\ *Corresponding author
}

\section{A B S T R A C T}

The present study were carried out in the Department of Genetics and Plant Breeding, N. D. University of Agriculture and Technology, Kumarganj Faizabad during Rabi season 2014-15 and 2015-16 entitled "Standardization of hydropriming duration for enhance seed

Keywords

Chickpea, Biofertilizers, Priming.

Article Info

Accepted:

14 July 2017

Available Online:

10 September 2017 yield and its quality parameters in chickpea (Cicer arietinum L.)" The objective of the study was to standardize the hydro priming duration Seed priming is a simple and cheap method but is acceptable in different areas. In order to the effect of hydropriming treatment on germination percentage in caraway (Carum carviL.), this experiment was conducted by randomized block design (Factorial) design with three replications. The factor was including hydro priming $13\left(\mathrm{P}_{1}\right), 16\left(\mathrm{P}_{2}\right)$, and $18\left(\mathrm{P}_{3}\right)$ hours. The results showed that the effect of hydropriming was significant on germination percentage, seedling length, and seedling vigour index. Mean comparison showed that the highest germination percentage, seedling length were achieved by $\mathrm{P}_{2}$ and also the highest seedling dry weight and seedling vigour were achieved by $\mathrm{P}_{2}$ and lowest germination percentage, seedling length, seedling dry weight and seedling vigour were achieved by $\mathrm{P}_{1}$. The results of this experiment showed that hydro priming method can improve the germination percentage in field condition and lab condition.

\section{Introduction}

Chickpea (Cicer arietinum L.) is the most important pulse crop in India with an average yield of $1500-2000 \mathrm{~kg} / \mathrm{ha}$. Chickpea is a diploid species with a chromosome number $2 \mathrm{n}=16$. It belongs to subfamily Papilionaceae of the family Leguminaceae. It's seeds contain $16.4-31.2 \%$ protein, $3.0 \%$ fibre, $38.1-73.3 \%$ carbohydrates, $1.6-9.0 \%$ cellulose, $0.2 \% \mathrm{Ca}, 0.3 \% \mathrm{P}, 3.0 \%$ ash, vitamins $(\mathrm{C}$ and $\mathrm{B})$ and minerals $(\mathrm{Mg}, \mathrm{Zn}, \mathrm{K}$, $\mathrm{Fe}$ ) (Huda et al., 2003 and Ozer et al., 2010). Combined inoculation of Rhizobium with Pseudomonas striata or Bacillus polymyxa and with Bacillus megaterium have shown increased dry matter, grain yield and phosphorus uptake significantly over the uninoculated control in legumes (Elkoca et al., 2008). Phosphate solubilising bacteria (PSB) plays an important role in making phosphorus available to crop plants.

The present study was undertaken comparative study of biofertilizers and seed priming on seed yield and quality of chickpea (Cicer arietinum L.). Seed priming, several processes including storage, material 
handling, activation and synthesis of a number of enzymes and nucleic acids, repair and build up, Several biochemical and physiological changes have been observed in seeds during ageing membrane disruption is one of the main reason of seed deterioration the major cause of membrane disruption are increase in free fatty acid level and free radical productivity by lipid peroxydation. Different techniques could be used to enhance crop yield. Priming is also thought to increase free radical scavenging enzymes activity and counteract the effect of lipid peroxidation and reduce leakage of metabolites. The early improvements may increase the rate of uniformity of seed germination and seedling emergence. (Afzal et al., 2002)

Hydropriming (using distilled water with temperature of $30^{\circ} \mathrm{C}$ for 18 days) on chickpea germination under 3 different temperatures of 15,25 , and $38^{\circ} \mathrm{C}$, coming to conclusion that no significant impact of osmopriming and hydro-priming would be found between 25 and $38^{\circ} \mathrm{C}$ on seed germination. The beneficial effects of osmo- and hydropriming are associated with increase in radicle and plumule lengths. Chickpea is a short-season edible grain legume grown crop, which cultivated mostly in arid and semi-arid region of the world where salinity, high temperature, rapid soil drying, and crust formation are barriers to good chickpea crop establishment. However, no study has been previously reported in the context of Iran on the effects of different osmopriming on chickpea seed germination and early seedling growth. Consequently, the aim of this study was to elucidate the effects of osmo-priming on seed emergence and early seedling growth of chickpea under the pot condition (Nascimento, 2003).

\section{Materials and Methods}

The field experiments under present investigation were conducted during Rabi
2014-15 and 2015-16 at Student Instructional Farm and lab experiments were carried out in Seed Testing Laboratory of Seed Technology Section, N. D. University of Agriculture and Technology, Kumarganj, Faizabad (U. P.). Geographically, Narendra Nagar situated between $26.47^{0} \mathrm{~N}$ latitude, $82.12^{0}$ longitude and at an altitude of 113 meters above the mean sea level.

The climate of district Faizabad is semi-arid with hot summer and cold winter. Nearly 80 per cent of total rain fall is received during the monsoon the treatment details are presented in table 1 .

Plant to plant distance and row to row distance was 10 and $30 \mathrm{~cm}$, respectively. Fertilizer was applied @ 20:40:40 ( $\left.\mathrm{kg} \mathrm{ha}^{-1}\right)$ $\mathrm{N}: \mathrm{P}: \mathrm{K}$ at the time of sowing. The chickpea crop was cultivated using standard agronomic practices. The observations were recorded at days to plant height $(\mathrm{cm})$, number of branches per plant, $50 \%$ flowering, number of pods per plant, number of seeds per pod, biological yield per plant $(\mathrm{g})$, seed index $(\mathrm{g})$, harvest index $(\%)$,seed yield per plant $(\mathrm{g})$, seed yield (q/ha)

\section{Result and Discussion}

\section{Mean performance}

The data presented in table 2, show mean performance of 27 treatments for 16 characters. The grand mean and range for all the traits are also depicted in table 2 .

\section{Days to $50 \%$ flowering}

Scanning of data presented in table 1 indicates that hydro-priming of seed influenced the flowering period of chickpea and minimum flowering period of 96.70 and 97.61 days was associated with $13 \mathrm{hrs}$ and $16 \mathrm{hrs}$ hydropriming during 2014-15 and 2015-16 respectively. During 2014-15 flowering 
periods were statistically similar in $16 \mathrm{hrs}$ and $18 \mathrm{hrs}$ priming. While during 2015-16 similar flowering period was noted in 13, 16 and 18 hrs priming of seeds.

\section{Days to maturity}

Scanning of data presented in table 1 indicates that hydroprimied seed influenced the flowering period of chickpea. Minimum flowering period 146.5 and 145.7 days were associated with 16 hours and 18 hours hydro priming during 2014-15 and 2015-16 respectively. During 2014-15 maturity periods were statistically similar in 13,16 and $18 \mathrm{hrs}$ priming while during 2015-16 similar maturity period was noted in 13,16 , and 18 hrs priming of seeds.

\section{Plant height (cm) at 30 DAS}

Scanning of data presented in table 2 showed due to that hydropriming of seed influenced the plant height of chickpea the maximum plant height of 10.16 and $11.14 \mathrm{~cm}$ was associated with 16 hours priming during season first and season second respectively. During season first plant height was statistically similar in $18 \mathrm{hrs}$ priming. 16 hours hydroprimed, priming seed was significantly superior to 13 and $18 \mathrm{hrs}$ were recorded in season second.

\section{Plant height $(\mathrm{cm})$ at 60 DAS}

Scanning of data presented in table 2 indicates that plant height of chickpea affected by hydro priming technique. Maximum plant height 18.50 and $18.13 \mathrm{~cm}$ was associated with 16 hrs hydro priming during 2014-15 and 2015-16 respectively. During 2014-15 plant height was statistically at par with 18 hrs priming. While during 2015-16, plant height in 16 hrs was statistically at par with 13 and $18 \mathrm{hrs}$ priming of seeds.

\section{Number of branches per plant at 30 DAS}

Table 3 indicates that hydro priming of seed influenced the number of branches per plant in chickpea and maximum branches 5.97 and 6.52 was associated with 16 hrs hydro priming during 2014-15 and 2015-16 respectively. During 2014-15 numbers of branches in $16 \mathrm{hrs}$ were significantly superior to 13 and $18 \mathrm{hrs}$ priming. While during 201516 numbers of branches were significantly superior with 13 and 18 hrs primed of seeds of chickpea variety (Pant G-186).

\section{Number of branches per plant at 60 DAS}

Scanning of data presented in table 3 indicates that hydro priming of seed influenced the number of branches per plant of chickpea and maximum branches 10.20 and 10.71 were associated with $16 \mathrm{hrs}$ hydro priming during 2014-15 and 2015-16 on 60 days, respectively. During 2014-15 number of branches per plant in $16 \mathrm{hrs}$ was hydro priming significantly superior than 13 and 18 hrs. While during 2015-16 number of branches in $16 \mathrm{hrs}$ were significantly superior with $13 \mathrm{hrs}$ priming of seeds.

The increase in number of branches per plant could be due to atmospheric $\mathrm{N}$ fixed by Rhizobium and growth promoting substances produced by P-solubilizers. These results are in collaboration with the earlier finding in chickpea (Jain et al., 1999).

\section{Number of pods per plant}

Scanning of data in table 4 indicates that hydro priming influenced the number of pods per plant of chickpea and maximum pod 76.69 and 72.19 were associated with 16 hour hydro priming during 2014-15 and 2015-16 respectively. During 2014-15 numbers of pods per pod in $16 \mathrm{hrs}$ were significantly superior to 13 and $18 \mathrm{hrs}$ priming. 
Table.1

\begin{tabular}{|c|c|c|c|c|c|c|c|c|c|c|c|c|c|}
\hline \multirow[t]{3}{*}{ S.N. } & \multirow{3}{*}{ Characters } & \multicolumn{6}{|c|}{ Days to $50(\%)$ flowering } & \multicolumn{6}{|c|}{ Days to maturity } \\
\hline & & \multicolumn{3}{|c|}{ Rabi 2014-15 } & \multicolumn{3}{|c|}{ Rabi 2015-16 } & \multicolumn{2}{|c|}{ Rabi 2014-15 } & \multicolumn{4}{|c|}{ Rabi 2015-16 } \\
\hline & & $\begin{array}{l}\text { HP13 } \\
\text { Hrs. }\end{array}$ & $\begin{array}{l}\text { HP16 } \\
\text { Hrs. }\end{array}$ & $\begin{array}{l}\text { HP18 } \\
\text { Hrs. }\end{array}$ & $\begin{array}{l}\text { HP13 } \\
\text { Hrs. }\end{array}$ & $\begin{array}{l}\text { HP16 } \\
\text { Hrs. }\end{array}$ & $\begin{array}{l}\text { HP18Hr } \\
\text { s. }\end{array}$ & $\begin{array}{l}\text { HP13 } \\
\text { Hrs. }\end{array}$ & HP16 Hrs. & $\begin{array}{l}\text { HP18 } \\
\text { Hrs. }\end{array}$ & $\begin{array}{l}\text { HP13 } \\
\text { Hrs. }\end{array}$ & $\begin{array}{l}\text { HP16 } \\
\text { Hrs. }\end{array}$ & $\begin{array}{l}\text { HP18 } \\
\text { Hrs. }\end{array}$ \\
\hline \multirow[t]{2}{*}{$\mathbf{T}_{1}$} & Trichoderma & & & & & & & & & & & & \\
\hline & $5 \mathrm{~g}$ & 94.00 & 102.33 & 96.67 & 92.67 & 101.33 & 96.33 & 150.33 & 148.00 & 147.33 & 150.00 & 145.67 & 147.00 \\
\hline \multirow[t]{2}{*}{$\mathbf{T}_{2}$} & Trichoderma & & & & & & & & & & & & \\
\hline & $7.5 \mathrm{~g}$ & 91.00 & 105.67 & 97.00 & 94.00 & 98.13 & 99.67 & 151.67 & 149.67 & 145.33 & 150.67 & 149.33 & 142.00 \\
\hline \multirow[t]{2}{*}{$\mathbf{T}_{3}$} & Trichoderma & & & & & & & & & & & & \\
\hline & $10 \mathrm{~g}$ & 95.67 & 97.00 & 94.33 & 106.33 & 99.00 & 104.00 & 146.33 & 147.33 & 144.67 & 144.33 & 147.67 & 143.67 \\
\hline $\mathbf{T}_{4}$ & PSB $7.5 \mathrm{~g}$ & 101.67 & 94.33 & 98.67 & 101.67 & 100.33 & 97.67 & 144.67 & 146.00 & 146.67 & 144.33 & 144.67 & 144.67 \\
\hline $\mathbf{T}_{5}$ & PSB $10 \mathrm{~g}$ & 97.67 & 97.33 & 102.00 & 102.00 & 96.33 & 99.00 & 145.00 & 143.67 & 144.67 & 144.67 & 144.67 & 146.00 \\
\hline$T_{6}$ & PSB $12.5 \mathrm{~g}$ & 104.67 & 96.00 & 106.00 & 99.67 & 93.67 & 104.33 & 153.67 & 149.67 & 153.00 & 151.33 & 147.67 & 148.67 \\
\hline \multirow[t]{2}{*}{$\mathbf{T}_{7}$} & Rhizobium & & & & & & & & & & & & \\
\hline & $7.5 \mathrm{~g}$ & 96.67 & 99.33 & 94.67 & 96.67 & 94.00 & 105.33 & 145.33 & 143.33 & 149.00 & 146.67 & 144.00 & 148.67 \\
\hline \multirow[t]{2}{*}{$\mathbf{T}_{8}$} & Rhizobium & & & & & & & & & & & & \\
\hline & $10 \mathrm{~g}$ & 93.33 & 98.00 & 95.33 & 94.00 & 97.67 & 105.00 & 141.67 & 143.67 & 149.33 & 142.33 & 145.00 & 146.00 \\
\hline $\mathbf{T}_{9}$ & $\begin{array}{l}\text { Rhizobium } \\
12.5 \mathrm{~g}\end{array}$ & 95.67 & 93.33 & 100.33 & 96.33 & 98.00 & 100.67 & 143.33 & 148.00 & 146.67 & 141.00 & 144.00 & 144.67 \\
\hline \multicolumn{2}{|c|}{ Grand Mean } & 96.70 & 98.15 & 98.33 & 98.15 & 97.61 & 101.33 & 146.89 & 146.59 & 147.41 & 146.15 & 145.85 & 145.70 \\
\hline & SEM & & CD 5\% & CD $1 \%$ & & CD 5\% & CD 1\% & & CD 5\% & CD 1\% & & CD 5\% & CD $1 \%$ \\
\hline \multicolumn{2}{|c|}{ Due to Treatment } & 0.972 & 2.759 & 3.677 & 1.397 & 3.964 & 5.284 & 0.548 & 1.554 & 2.072 & 0.721 & 2.046 & 2.727 \\
\hline \multicolumn{2}{|c|}{ Due to Priming } & 0.561 & 1.593 & 2.123 & 0.807 & 2.288 & 3.050 & 0.316 & 0.897 & 1.196 & 0.416 & 1.181 & 1.574 \\
\hline \multicolumn{2}{|c|}{ Due to Interaction PxT } & 1.684 & 4.778 & 4.778 & 2.420 & 6.865 & 9.151 & 0.949 & 2.692 & 3.588 & 1.249 & 3.543 & 4.723 \\
\hline
\end{tabular}


Table.2

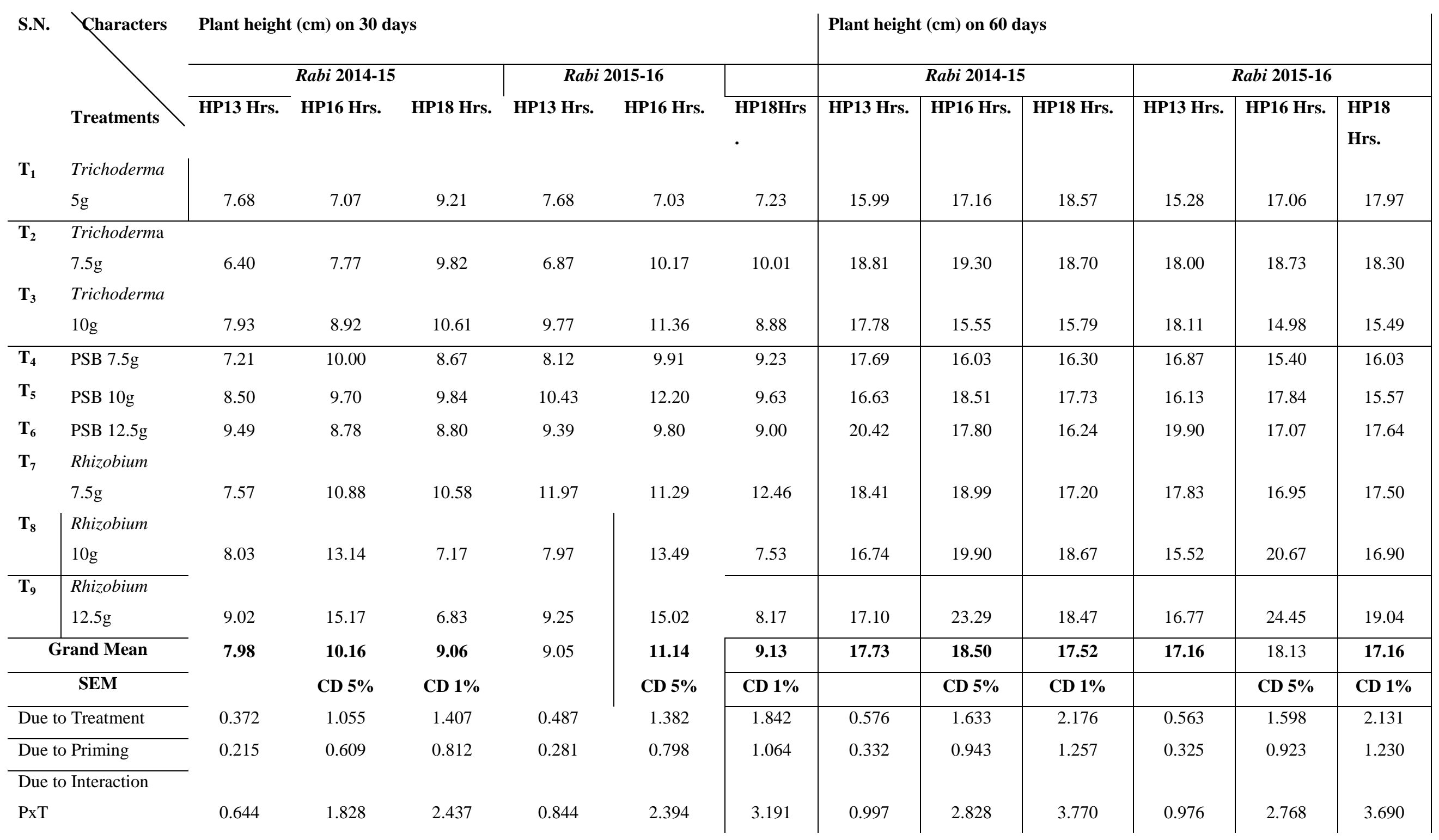


Table.3

\begin{tabular}{|c|c|c|c|c|c|c|c|c|c|c|c|c|c|}
\hline \multirow{3}{*}{$\begin{array}{l}\text { S.N } \\
\cdot \\
\mathbf{T}_{1}\end{array}$} & \multirow{3}{*}{ Characters } & \multicolumn{6}{|c|}{ Number of branches/plant on 30 days } & \multicolumn{6}{|c|}{ Number of branches/plant on 60 days } \\
\hline & & \multicolumn{3}{|c|}{ Rabi 2014-15 } & \multicolumn{3}{|c|}{ Rabi 2015-16 } & \multicolumn{3}{|c|}{ Rabi 2014-15 } & \multicolumn{3}{|c|}{ Rabi 2015-16 } \\
\hline & & $\begin{array}{l}\text { HP13 } \\
\text { Hrs. }\end{array}$ & HP16 Hrs. & HP18 Hrs. & HP13 Hrs. & HP16 Hrs. & $\begin{array}{l}\text { HP18H } \\
\text { rs. }\end{array}$ & $\begin{array}{l}\text { HP13 } \\
\text { Hrs. }\end{array}$ & HP16 Hrs. & $\begin{array}{l}\text { HP18 } \\
\text { Hrs. }\end{array}$ & HP13 Hrs. & HP16 Hrs. & HP18 Hrs. \\
\hline \multirow[t]{2}{*}{$\mathbf{T}_{2}$} & Trichoderma & & & & & & & & & & & & \\
\hline & $7.5 \mathrm{~g}$ & 4.60 & 5.67 & 4.27 & 4.13 & 5.53 & 5.13 & 8.07 & 9.60 & 8.93 & 9.27 & 8.63 & 9.70 \\
\hline \multirow[t]{2}{*}{$\mathbf{T}_{3}$} & Trichoderma & & & & & & & & & & & & \\
\hline & $10 \mathrm{~g}$ & 5.27 & 4.60 & 5.00 & 3.53 & 6.80 & 4.27 & 7.93 & 7.87 & 11.20 & 8.00 & 7.87 & 10.80 \\
\hline $\mathbf{T}_{4}$ & PSB $7.5 \mathrm{~g}$ & 4.73 & 5.67 & 5.40 & 4.07 & 5.47 & 5.40 & 8.93 & 9.37 & 8.40 & 9.67 & 10.27 & 8.67 \\
\hline $\mathbf{T}_{5}$ & PSB $10 \mathrm{~g}$ & 4.77 & 6.00 & 6.13 & 5.17 & 7.07 & 6.13 & 10.20 & 11.47 & 9.53 & 8.80 & 11.93 & 8.20 \\
\hline $\mathbf{T}_{6}$ & PSB $12.5 \mathrm{~g}$ & 4.67 & 5.40 & 5.53 & 4.00 & 5.73 & 4.40 & 8.80 & 9.13 & 10.40 & 7.73 & 10.60 & 11.33 \\
\hline $\mathbf{T}_{7}$ & Rhizobium & & & & & & & & & & & & \\
\hline & $7.5 \mathrm{~g}$ & 5.60 & 5.40 & 5.03 & 4.80 & 5.80 & 6.40 & 10.07 & 8.53 & 9.27 & 11.00 & 9.47 & 10.07 \\
\hline $\mathbf{T}_{8}$ & $\begin{array}{l}\text { Rhizobium } \\
10 \mathrm{~g}\end{array}$ & 4.63 & 7.07 & 4.53 & 4.60 & 7.13 & 4.80 & 9.53 & 12.67 & 10.47 & 8.40 & 12.43 & 9.00 \\
\hline $\mathbf{T}_{9}$ & $\begin{array}{l}\text { Rhizobium } \\
12.5 \mathrm{~g}\end{array}$ & 6.53 & 8.13 & 5.00 & 5.93 & 8.33 & 4.87 & 8.87 & 14.53 & 8.40 & 9.47 & 14.97 & 13.60 \\
\hline \multicolumn{2}{|c|}{ Grand Mean } & 5.07 & 5.97 & 5.05 & 4.57 & 6.52 & 5.09 & 8.99 & 10.20 & 9.41 & 8.99 & 10.71 & 10.00 \\
\hline & SEM & & CD 5\% & CD 1\% & & CD 5\% & CD 1\% & & CD 5\% & CD $1 \%$ & & CD 5\% & CD 1\% \\
\hline \multicolumn{2}{|c|}{ Due to Treatment } & 0.264 & 0.748 & 0.997 & 0.223 & 0.634 & 0.845 & 0.395 & 1.121 & 1.495 & 0.434 & 1.231 & 1.640 \\
\hline \multicolumn{2}{|c|}{ Due to Priming } & 0.152 & 0.432 & 0.576 & 0.129 & 0.366 & 0.488 & 0.228 & 0.647 & 0.863 & 0.250 & 0.710 & 0.947 \\
\hline \multicolumn{14}{|c|}{ Due to Interaction } \\
\hline PxT & & 0.457 & 1.295 & 1.727 & 0.387 & 1.097 & 1.463 & 0.685 & 1.942 & 2.589 & 0.751 & 2.131 & 2.841 \\
\hline
\end{tabular}




\section{Table.4}

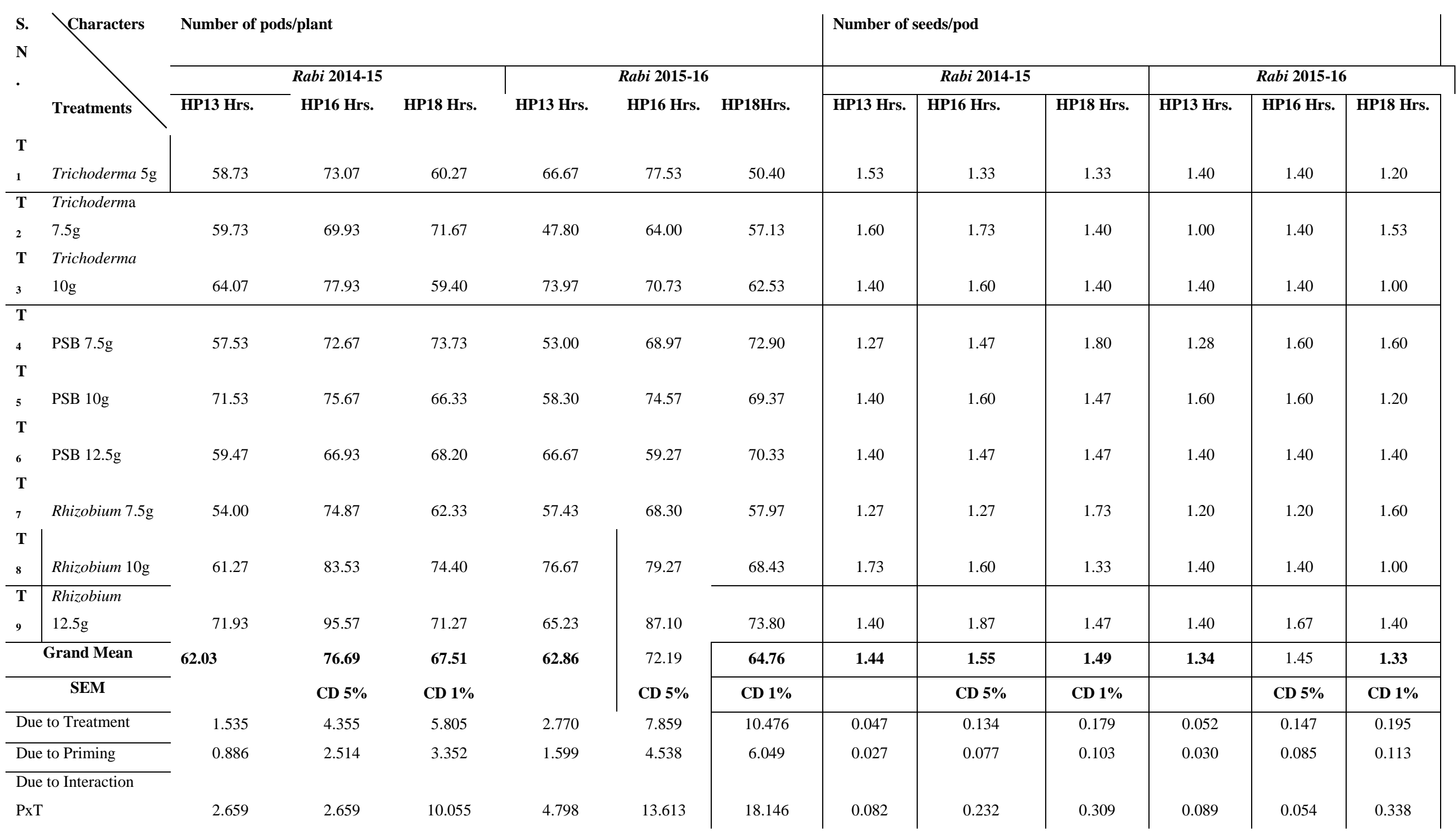


Table.5

\begin{tabular}{|c|c|c|c|c|c|c|c|c|c|c|c|c|c|}
\hline \multirow[t]{3}{*}{ S. } & Characters & \multicolumn{6}{|c|}{ Biological yield (g) } & \multicolumn{6}{|c|}{ Harvest index } \\
\hline & & \multicolumn{3}{|c|}{ Rabi 2014-15 } & \multicolumn{3}{|c|}{ Rabi 2015-16 } & \multicolumn{3}{|c|}{ Rabi 2014-15 } & \multicolumn{3}{|c|}{ Rabi 2015-16 } \\
\hline & Treatments & HP13 Hrs. & HP16 Hrs. & HP18 Hrs. & HP13 Hrs. & HP16 Hrs. & HP18Hrs. & HP13 Hrs. & HP16 Hrs. & HP18 Hrs. & HP13 Hrs. & HP16 Hrs. & $\begin{array}{l}\text { HP18 } \\
\text { Hrs. }\end{array}$ \\
\hline $\begin{array}{l}T \\
1\end{array}$ & Trichoderma $5 \mathrm{~g}$ & 48.67 & 58.60 & 50.33 & 46.57 & 53.50 & 45.53 & 31.52 & 39.65 & 31.19 & 31.19 & 30.08 & 25.30 \\
\hline $\begin{array}{l}\mathrm{T} \\
2 \\
\mathrm{~T}\end{array}$ & $\begin{array}{l}\text { Trichoderma } \\
7.5 \mathrm{~g} \\
\text { Trichoderma }\end{array}$ & 52.53 & 56.53 & 51.44 & 45.60 & 50.90 & 52.97 & 38.39 & 38.37 & 26.07 & 30.49 & 27.39 & 32.59 \\
\hline 3 & $10 \mathrm{~g}$ & 56.40 & 53.44 & 46.93 & 47.93 & 51.57 & 51.77 & 27.66 & 30.77 & 31.77 & 34.70 & 30.99 & 34.24 \\
\hline $\begin{array}{l}T \\
4 \\
T\end{array}$ & PSB 7.5g & 44.53 & 63.47 & 50.00 & 49.03 & 56.13 & 60.30 & 41.24 & 27.00 & 33.66 & 37.37 & 36.45 & 31.19 \\
\hline $\begin{array}{l}5 \\
T\end{array}$ & PSB 10g & 44.63 & 59.87 & 52.10 & 47.00 & 49.37 & 47.73 & 30.65 & 35.85 & 41.94 & 42.71 & 33.06 & 30.14 \\
\hline $\begin{array}{l}6 \\
T\end{array}$ & PSB $12.5 \mathrm{~g}$ & 53.33 & 50.57 & 55.80 & 57.47 & 50.00 & 49.13 & 27.38 & 26.94 & 36.29 & 30.24 & 35.41 & 27.81 \\
\hline $\begin{array}{l}7 \\
T\end{array}$ & Rhizobium $7.5 \mathrm{~g}$ & 61.73 & 48.03 & 52.87 & 48.70 & 59.47 & 52.10 & 32.81 & 28.06 & 31.45 & 33.34 & 30.03 & 38.22 \\
\hline 8 & Rhizobium $10 \mathrm{~g}$ & 44.13 & 53.63 & 42.70 & 54.80 & 61.37 & 55.50 & 45.93 & 46.15 & 37.63 & 29.04 & 38.80 & 30.40 \\
\hline $\begin{array}{l}\text { T } \\
9\end{array}$ & $\begin{array}{l}\text { Rhizobium } \\
12.5 \mathrm{~g}\end{array}$ & 49.20 & 75.73 & 55.40 & 58.23 & 64.00 & 54.00 & 41.41 & 42.78 & 33.41 & 33.56 & 44.56 & 38.56 \\
\hline & Grand Mean & 50.57 & 57.76 & 50.84 & 50.59 & 55.14 & 52.11 & 35.22 & 35.06 & 33.71 & 33.63 & 34.08 & 32.05 \\
\hline & SEM & & CD 5\% & CD 1\% & & CD 5\% & CD 1\% & & CD 5\% & CD 1\% & & CD 5\% & CD 1\% \\
\hline & e to Treatment & 1.743 & 4.944 & 6.590 & 1.762 & 4.997 & 6.662 & 1.461 & 4.145 & 5.526 & 1.921 & 5.450 & 7.265 \\
\hline & to Priming & 1.006 & 2.854 & 3.805 & 1.017 & 2.885 & 3.846 & 0.844 & 2.393 & 3.190 & 1.109 & 3.147 & 4.195 \\
\hline & e to Interaction & & & & & & & & & & & & \\
\hline Pxt & & 3.018 & 8.563 & 11.414 & 3.051 & 8.656 & 11.538 & 2.531 & 7.180 & 9.571 & 3.328 & 9.440 & 12.584 \\
\hline
\end{tabular}


Table.6

\begin{tabular}{|c|c|c|c|c|c|c|c|c|c|c|c|c|c|}
\hline \multirow[t]{2}{*}{ s. } & Characters & \multicolumn{6}{|c|}{ Seed yield/Plant (g) } & \multicolumn{6}{|c|}{ Seed yield (Kg/ha) } \\
\hline & & \multicolumn{3}{|c|}{ Rabi 2014-15 } & \multicolumn{3}{|c|}{ Rabi 2015-16 } & \multicolumn{2}{|c|}{ Rabi 2014-15 } & \multicolumn{4}{|c|}{ Rabi 2015-16 } \\
\hline $\mathbf{T}$ & Treatments & HP13 Hrs. & HP16 Hrs. & HP18 Hrs. & HP13 Hrs. & HP16 Hrs. & HP18Hrs. & HP13 Hrs. & HP16 Hrs. & HP18 Hrs. & HP13 Hrs. & HP16 Hrs. & HP18 Hrs. \\
\hline 1 & Trichoderma $5 \mathrm{~g}$ & 15.21 & 23.20 & 15.67 & 14.40 & 16.03 & 11.50 & 1741.27 & 1607.53 & 1545.07 & 1772.00 & 1450.63 & 1455.03 \\
\hline $\mathbf{T}$ & $\begin{array}{l}\text { Trichoderma } \\
7.5 \mathrm{~g} \\
\text { Trichoderma }\end{array}$ & 20.13 & 21.67 & 13.31 & 13.87 & 13.97 & 16.83 & 1544.63 & 1549.03 & 1720.33 & 1581.53 & 1860.83 & 1746.90 \\
\hline 3 & $10 \mathrm{~g}$ & 15.68 & 16.41 & 14.90 & 16.63 & 15.97 & 17.63 & 1747.67 & 1845.90 & 1756.93 & 1825.10 & 2005.50 & 1596.67 \\
\hline $\begin{array}{l}T \\
4 \\
T\end{array}$ & PSB $7.5 \mathrm{~g}$ & 18.20 & 16.90 & 16.80 & 18.23 & 20.03 & 18.83 & 1682.07 & 1779.23 & 2142.40 & 1433.50 & 1740.73 & 1627.00 \\
\hline $\begin{array}{l}5 \\
T\end{array}$ & PSB $10 \mathrm{~g}$ & 13.63 & 21.30 & 21.73 & 20.07 & 16.30 & 14.30 & 1834.97 & 2138.57 & 1728.83 & 1625.97 & 2165.47 & 1761.73 \\
\hline $\begin{array}{l}6 \\
T\end{array}$ & PSB $12.5 \mathrm{~g}$ & 14.57 & 13.63 & 19.90 & 17.33 & 17.77 & 13.63 & 1655.10 & 1987.47 & 1813.43 & 2149.83 & 1692.70 & 1651.33 \\
\hline $\begin{array}{l}7 \\
T\end{array}$ & Rhizobium $7.5 \mathrm{~g}$ & 20.17 & 13.40 & 16.70 & 16.40 & 17.70 & 19.93 & 2137.23 & 2017.27 & 2355.73 & 1474.77 & 1757.37 & 1849.07 \\
\hline 8 & Rhizobium 10g & 20.13 & 24.73 & 16.00 & 15.90 & 23.57 & 16.97 & 2453.03 & 2284.63 & 1650.37 & 1610.67 & 2247.07 & 1463.70 \\
\hline $\mathbf{T}$ & $\begin{array}{l}\text { Rhizobium } \\
12.5 \mathrm{~g}\end{array}$ & 20.05 & 32.40 & 18.37 & 18.97 & 28.53 & 20.30 & 1752.87 & 2579.97 & 2046.47 & 1755.77 & 2477.03 & 2016.70 \\
\hline & Grand Mean & 17.53 & 20.40 & 17.04 & 16.87 & 18.87 & 16.66 & 1838.76 & 1976.62 & 1862.17 & 1692.13 & 1933.04 & 1685.35 \\
\hline & SEM & & CD 5\% & CD $1 \%$ & & CD 5\% & CD 1\% & & CD 5\% & CD 1\% & & CD 5\% & CD 1\% \\
\hline & to Treatment & 0.648 & 1.839 & 2.451 & 0.875 & 2.482 & 3.309 & 50.371 & 142.897 & 190.482 & 41.339 & 117.274 & 156.326 \\
\hline & to Priming & 0.374 & 1.062 & 1.415 & 0.505 & 1.433 & 1.910 & 29.081 & 82.502 & 109.975 & 23.867 & 67.708 & 90.255 \\
\hline & to Interaction & & & & & & & & & & & & \\
\hline Px & & 1.123 & 3.185 & 4.245 & 1.515 & 4.299 & 5.731 & 87.244 & 247.505 & 329.924 & 71.600 & 203.124 & 270.765 \\
\hline
\end{tabular}


Table.7

S. Characters

100-Seed weight (g)

Germination (\%)

\section{Rabi 2014-15}

Rabi 2014-15

Rabi 2015-16

Hrs.

HP16 Hrs.

\begin{tabular}{|l|l|l|}
\hline \multicolumn{2}{c}{ Rabi 2015-16 } \\
\hline HP18 Hrs. & HP13 Hrs. & HP16 Hrs.
\end{tabular}

\begin{tabular}{l|l} 
rs. & HP18 Hrs.
\end{tabular}

$\mathbf{T}$ Treatments

T Trichoderma

$2 \quad 7.5 \mathrm{~g}$

\begin{tabular}{l}
$18.91 \quad 18.60$ \\
\hline
\end{tabular}

$17.80 \quad 20.90$

\begin{tabular}{l|l}
19.05 & 86.33
\end{tabular}

91.42

T Trichoderma

$$
3
$$$$
21.10
$$

$\begin{array}{lll}18.75 & 18.53 & 20.60\end{array}$

18.93

$18.47 \quad 20.49$

19.02

18.23

18.37

20.70

$21.03 \quad 20.90$

20.90

19.10

19.17

21.20

PSB $10 \mathrm{~g}$

17.30

19.93

20.40

20.90

$\quad 89.50$

T

T

\begin{tabular}{l|l}
$\mathbf{8}$ & Rhizobium 10g \\
\hline $\mathbf{T}$ & Rhizobium
\end{tabular}

$\begin{array}{ll}9 & 12.5 \mathrm{~g}\end{array}$ Grand Mean

16.9

16.93
$\mathbf{1 9 . 2 2}$

Due to Treatment

0.360

Due to Priming

0.208

Due to Interaction

PxT

0.623

19.89

19.37

20.73

20.07

21.53

$19.50 \quad 20.10$

20.90

17.73

19.70

(1)

(17.89

19.20

$\begin{array}{lll}20.10 & 17.50 & 20.20\end{array}$

$\begin{array}{ll}19.45 & 18.87\end{array}$

19.86

CD 5\% CD 1\%

$1.020 \quad 1.360$

0.439

0.589

0.785

0.253

$1.767 \quad 2.355$

0.760

20.47
$\mathbf{1 9 . 7 9}$
CD 5\%
1.245
0.719
2.156

\begin{tabular}{|c|c|c|c|}
\hline 16.88 & 89.75 & 88.92 & 85.83 \\
\hline 19.00 & 87.75 & 88.25 & 87.40 \\
\hline $\mathbf{1 9 . 6 6}$ & $\mathbf{8 8 . 1 4}$ & $\mathbf{8 8 . 0 1}$ & $\mathbf{8 6 . 7 9}$ \\
\hline CD 1\% & & $\mathbf{C D ~ 5 \%}$ & $\mathbf{C D ~ 1 \%}$ \\
\hline 1.659 & 0.646 & 1.833 & 2.443 \\
0.958 & 0.373 & 1.058 & 1.410 \\
2.873 & 1.119 & 3.174 & 4.231
\end{tabular}


Int.J.Curr.Microbiol.App.Sci (2017) 6(9): 665-679

Table.8

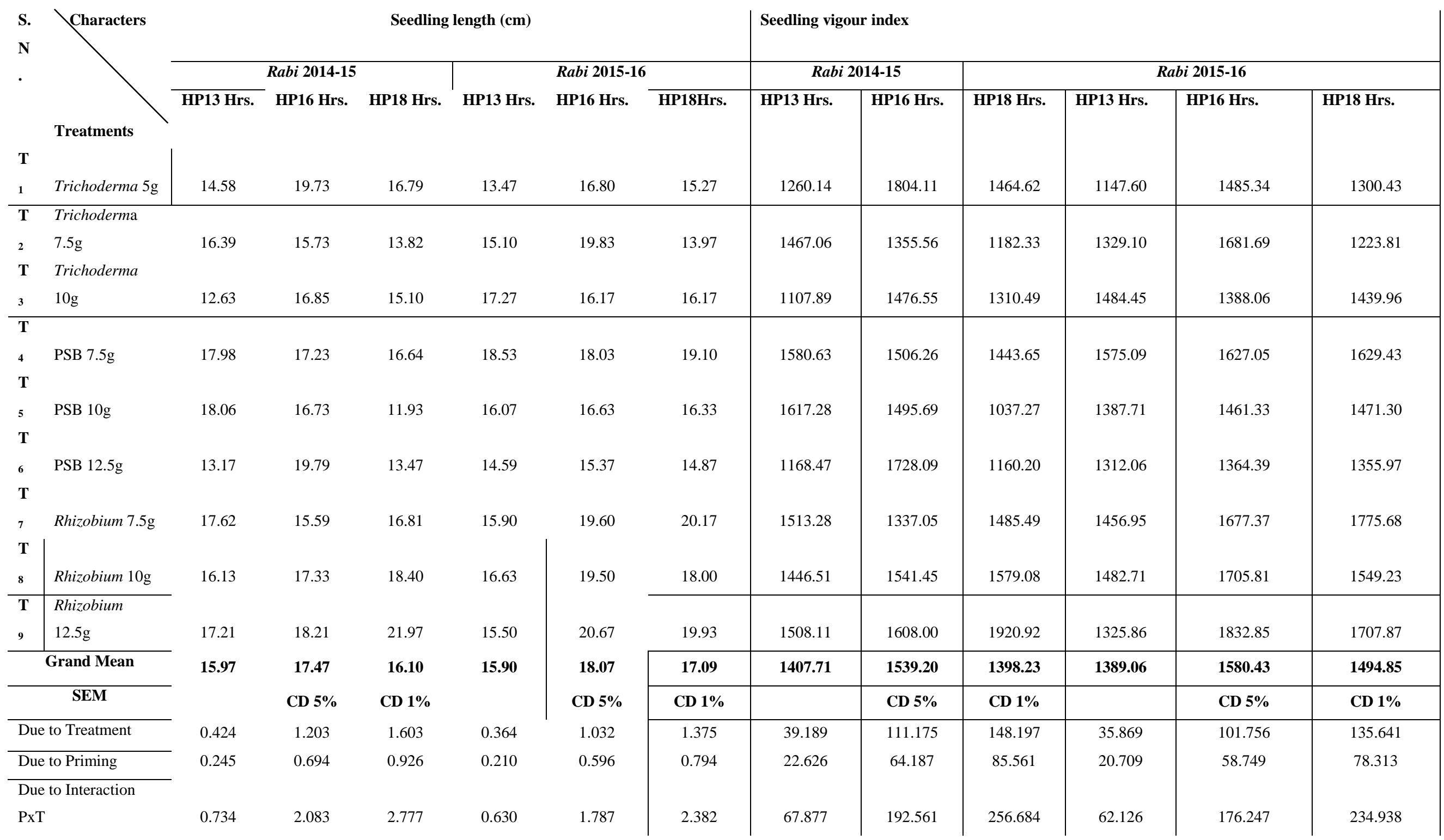




\section{Treatments used in this study}

\begin{tabular}{|c|l|}
\hline Treatment No. & Description \\
\hline $\mathrm{P}_{1}$ & Hydro priming with distilled water $13 \mathrm{hrs}$ \\
\hline $\mathrm{P}_{2}$ & Hydro priming with distilled water $16 \mathrm{hrs}$ \\
\hline $\mathrm{P}_{3}$ & Hydro priming with distilled water $18 \mathrm{hrs}$ \\
\hline $\mathrm{T}_{1}$ & Trichoderma $5 \mathrm{gm}$ \\
\hline $\mathrm{T}_{2}$ & Trichoderma $7.5 \mathrm{gm}$ \\
\hline $\mathrm{T}_{3}$ & Trichoderma $10 \mathrm{gm}$ \\
\hline $\mathrm{T}_{4}$ & PSB $7.5 \mathrm{gm}$ \\
\hline $\mathrm{T}_{5}$ & PSB10 gm \\
\hline $\mathrm{T}_{6}$ & PSB $12.5 \mathrm{gm}$ \\
\hline $\mathrm{T}_{7}$ & Rhizobium $7.5 \mathrm{gm}$ \\
\hline $\mathrm{T}_{8}$ & Rhizobium $10 \mathrm{gm}$ \\
\hline $\mathrm{T}_{9}$ & Rhizobium $12.5 \mathrm{gm}$ \\
\hline
\end{tabular}

While during 2015-16 number of pods per plant in $16 \mathrm{hrs}$ was significantly superior to 13 and 18 hrs priming of seeds.

The effects of organic and biologic fertilizers on soybean growth and quality of seed, Mekki and Amel (2005) showed that the number of pods per plant was increased by applying biofertilizer.

\section{Number of seeds per pod}

Revealed table 4 hydro priming of seed influenced the number of seeds per pod in chickpea and maximum seeds per pod 1.55 and 1.45 were associated with 16 hours hydro priming during 2014-15 and 2015-16 both seasons, respectively.

During 2014-15 numbers of seeds per pod in $16 \mathrm{hrs}$ were statistically similar with 13 and 18 hrs priming. Besides during 2015-16 numbers of seeds per pod in $16 \mathrm{hrs}$ were statistically similar with 13 and 18 hrs priming of seeds. These results are in confirmation with that of Karadavut and Ozdemir (2001) and Fatima et al., 2008 who reported that inoculation significantly increased grain yield (20\% higher than control).

\section{Biological yield per plant (g)}

Scanning of data presented in table 5 indicates that hydro priming of seed influenced the biological yield per plant of chickpea and maximum biological yield per plant $57.76 \mathrm{~g}$ and $55.14 \mathrm{~g}$ were associated with 16 hours hydro priming during Rabi 2014-15 and 2015-16, respectively. During first season and second season biological yield per plant for $16 \mathrm{hrs}$ was significantly superior to 13 and 18 hrs priming period.

\section{Harvest index}

Scanning of data presented in table 5 indicates that hydro priming of seed influenced the harvest index of chickpea and maximum harvest index 35.2 and 34.08 were associated with 13 hours and 16 hours hydro priming during 2014-15 and 2015-16, respectively.

\section{Seed yield per plant (gm)}

Scanning of data presented in table 6 indicates that hydro priming of seed influenced the seed yield per plant of chickpea and the maximum seed yield per plant 20.40 and 18.87 were associated with 16 hours hydro priming duration in both years. During first year seed 
yield per plant for $16 \mathrm{hrs}$ was significantly superior to 13 and $18 \mathrm{hrs}$ priming time. While during second year seed yield per plant for 13 hrs priming time was significantly superior to 16 and $18 \mathrm{hrs}$ priming duration.

Bacteria had beneficial effect on plant growth and seed yield, because they fix atmospheric nitrogen and release auxins to the root zone to enhance growth (Rees et al., 2009). Addition of biofertilizer promotes bacterial response to nitrogen fixation and soil fertility. Higher rates of atmospheric nitrogen fixation promote growth and yield (El-Desuki et al., 2010).

\section{Seed yield (Kg/ha)}

The maximum seed yield 1976.62 and 1933.04 (Kg/ha) were associated with 16 hours hydropriming during both period respectively. During 2014-15 seed yield (Kg/ha) for $16 \mathrm{hrs}$ was significantly superior to 13 and $18 \mathrm{hrs}$ priming time. While during 2015-16 Seed yield (Kg/ha) in16 hrs was significantly superior than 13 and $18 \mathrm{hrs}$ priming period for chickpea seed crop production

It might be due to the availability of plant nutrients in the vicinity of rhizosphere and less losses of nutrient due to fertilizer banding. These results are in conformity with those of Din et al., (1999) who recorded maximum yield in band placement. Seed inoculation also significantly affected the grain yield of chickpea.

\section{0-seed weight $(\mathrm{g})$}

Revealed of data presented in table 7 indicates that hydro priming of seed influenced the 100 -seed weight of chickpea. The maximum 100-seed weight 19.45 and $19.86 \mathrm{~g}$ were associated with 16 and 13 hours hydro priming during 2014-15. During 2014-15, 100-seed weight in 16 hrs was statistically similar with $13 \mathrm{hrs}$ and $18 \mathrm{hrs}$ priming. However during 2015-16 similar 100 -seed weight was statistically similar in 13, 16 and $18 \mathrm{hrs}$ priming. Period for above mentioned chapter

\section{Germination \%}

During 2014-15 germination in $13 \mathrm{hrs}$ was statistically similar in 16 and 18 hrs priming. While during 2015-16 germination were statistically similar in 13 and $16 \mathrm{hrs}$ priming of seeds

\section{Seedling length}

Table 8 data indicates that hydro priming of seeds influenced the seedling length of chickpea and maximum seedling length 17.47 $\mathrm{cm}$ and $18.07 \mathrm{~cm}$ were associated with 16 hours hydro priming during 2014-15 and 2015-16, respectively. During 2014-15 seedling length for $16 \mathrm{hrs}$ significantly superior than 13 and 18 hrs priming. While during 2015-16 seedling length $\mathrm{cm}$ in $18 \mathrm{hrs}$ was significantly superior in 13 and16 hrs priming of seeds.

\section{Vigour index}

Scanning of data presented in table 8 showed that hydro priming technique seed influenced the vigour index of chickpea crop seed and maximum vigour index 1539.2 and 1580.4 were associated with $16 \mathrm{hrs}$ hydropriming during both season. Vigour index seed quality parameters for $16 \mathrm{hrs}$ were significantly superior to 13 and $18 \mathrm{hrs}$ priming period for first and second year.

From the present study it was concluded that Seed priming has been used to improve germination, reduce seedling emergence time, and improve stand establishment and yield. The beneficial effects of priming have been 
demonstrated for many field crops. It is the best solution of germination related problems especially when crops are grown under unfavorable conditions. Many priming techniques have been evolved which are being utilized in many crops now days. It can enhance rates and percentage of germination and seedling emergence which ensure proper stand establishment under a wide range of environmental conditions.

\section{References}

Alagawadi, A. R., and Gaur, A. C. 1998. Association effect of Rhizobium and phosphate solubilizing bacteria on the yield and nutrient uptake of chickpea. Plant and Soil, 105:241-246.

Alam, M.J., A. R. M. Solaiman and A. J. M. S. Karim. 1999. Nutrient uptake yield attributes and protein content of Chickpea as influenced by some Rhizobium strains. Annals of Bangladesh Agriculture 9(2):131-138.

Alam, M.J., A.R.M. Solaiman and A.J.M.S. Karim. 1999. Nutrient uptake yield attributes and protein content of Chickpea as influenced by some Rhizobium strains. Annals of Bangladesh Agriculture 9(2):131-138.

D'souza, P.S., 1996. Efficacy of phosphate solubilizing biofertilizers with different sources and levels of phosphorous in chickpea. M.Sc. (Agri.) Thesis, University of Agriculture Science, Dharwad, Karnataka (India).

Dilip Kumar, S.B., I. Berggen and A.M. Martensson 2001. Potential for improving pea production by coinoculation with Pseudomonas fluorescence and Rhizobium. Plant and Soil, 229:25-34.

Din, J., Rashid, A. and Ahmed, A.I. 1999. Diagnosis and correlation of phosphorus deficiency in chickpea grown in rainfed calcareous soils of Pakistan. Journal of
Indian Soil Sci. 477(3):504-509.

El-Deshukhi, M., M. Hafiz. M, R. Mahmoud. A. and S. Abd El-Al, 2010. Effect of organic and biofertilizer on the plant growth. Green pod yield, quality of Pea. International Journal of Academic Research, 2(1):87-92.

El-Hadi, E.A., EL-Sheikh, E. 1999. Effect of Rhizobium inoculation and nitrogen fertilization on yield and protein contents of six chickpea cultivars in marginal soils under irrigation nutrient cycling in agro ecosystem. 54(1):57-63.

Elkoca, E., Kantar, F. and Sahin, F. 2008. Influence of nitrogen fixing and phosphate solubilizing bacteria on nodulation, plant growth and yield of chickpea. Journal of Plant Nutrition. 33:157-171.

Fatima, Z., A. Bano, R. Sial and Aslam, M. 2008. Response of chickpea to plant growth regulators on nitrogen fixation and yield. Pakistan Journal of Botany 40(5):2005-2013.

Giri, Nishita, and Joshi, N. C. 2010. Growth and yield response of chick pea (Cicer arietinum) to seed inoculation with Rhizobium spp. Nature and Science, 8(9).

Huda, S., Siddique, N.A., Khatun, N., Rahman, M.H. and Morshed, M. 2003. Regeneration of shoot from cotyledon derived callus of chickpea (Cicer arietinum L.). Pakistan journal of Biological Sciences, 6:1310-1313.

Jain, P.C., and Kushwaha, PS., Dhakal, US, Khan, H. and Trivedi, SM. 1999. Response of chickpea to phosphorus and biofertilizer. Legume Research, 22:241-244.

Karadavut, U., and Ozdemir. S. 2001. Effect of Rhizobium inoculation and nitrogen application on yield and yield characters of chickpea. Anadolu. 11 (1):14-22.

Kaur, S., Gupta, A.K. and Kaur, N. 2005. Seed Priming Increases Crop Yield 
Possibly by Modulating Enzymes of Sucrose Metabolism in Chickpea. Journal of Agronomy and Crop Science, 191: 81-87.

Kaur, S., Gupta, A.K. and Kaur, N. 2005. Seed priming increases crop yield possibly by modulating enzymes of sucrose metabolism in Chickpea. Journal of Agronomy and Crop Science 191:81-87.

Kennedy, I.R., Choudhury, A.T.M.A. and Kecskes, M.L. 2004. Non-symbiotic bacterial diazotrophs in crop-farming systems: Can their potential for plant growth promotion be better exploited. Soil Biology and Biochemistry 36:12291244.

Meena, K N., Pareek, R.G. and Jat, R.S. 2001. Effect of phosphorus and biofertilizers on yield and quality of chickpea. Annals of agriculture Research 22(3):388-390.

Mekki, B.B., and Amel, A.G. 2005. Growth, yield and seed quality of soybean as effected by organic, biofertilizer and its application. Agriculture and Biological Science, 1:320-324.

Ozer, S., Karakoy, T., Toklu, F., Baloch, S.F., Kilian, B. and Ozkan, H. 2010. Nutrition and physic chemical variation in Turkish, Kabuli chickpea (Cicer arietinum L.) land races. Euphytica, 175:237-249.

Rupela, O.P., 1987. Nodulation and nitrozen fixation in Chickpea. Wallingford, U.K: CAB International.196-206

Singh, R.S., and Yadav, M.K., 2008. Effect of phosphorus and biofertilizers on growth, yield and nutrient uptake of long duration Pigeon pea under rainfed condition. Journal of Food Legume, 21(1): 46-48.

Timmons, D.R., R.E. Burwell and Holt. R.F. 1973. Nitrogen and phosphorus losses in surface runoff from Agricultural land as influenced by placement of broad cost fertilizer. Water Resource Research, 9:658-667.

\section{How to cite this article:}

Sarvjeet, S.C. Vimal and Pankaj Kumar. 2017. Standardization of Hydropriming Duration for Enhance Seed Yield and Its Quality Parameters in Chickpea (Cicer arietinum L.). Int.J.Curr.Microbiol.App.Sci. 6(9): 665-679. doi: https://doi.org/10.20546/ijcmas.2017.609.082 